\title{
Greenberger-Horne-Zeilinger state protocols for fully connected qubit networks
}

\author{
Andrei Galiautdinov* \\ Department of Physics and Astronomy, University of Georgia, Athens, GA 30602 \\ Mark W. Coffey $\dagger^{\dagger}$ and Ron Deiotte \\ Department of Physics, Colorado School of Mines, Golden, CO 80401
}

(Dated: November 26, 2018)

\begin{abstract}
We generalize the recently proposed Greenberger-Horne-Zeilinger (GHZ) tripartite protocol [A. Galiautdinov, J. M. Martinis, Phys. Rev. A 78, 010305(R) (2008)] to fully connected networks of weakly coupled qubits interacting by way of anisotropic Heisenberg exchange $g(X X+Y Y)+\tilde{g} Z Z$. Our model adopted here differs from the more familiar Ising-Heisenberg chain in that here every qubit interacts with every other qubit in the circuit. The assumption of identical couplings on all qubit pairs allows an elegant proof of the protocol for arbitrary $N$. In order to further make contact with experiment, we study fidelity degradation due to coupling imperfections by numerically simulating the $N=3$ and $N=4$ cases. Our simulations indicate that the best fidelity at unequal couplings is achieved when (a) the system is initially prepared in the uniform superposition state (similarly to how it is done in the ideal case), and (b) the entangling time and the final rotations on each of the qubits are appropriately adjusted.
\end{abstract}

PACS numbers: 75.10.Jm, 03.67.Bg, 03.67.Lx, 85.25.-j

\section{INTRODUCTION}

In the rapidly developing field of quantum computing the concept of quantum entanglement is considered to be of greatest practical importance. Many important applications, such as quantum communication [1], secret sharing [2], open-destination teleportation [3], fault-tolerant computing [4, [5], and others [6], rely heavily on an architecture's ability to generate multipartite entangling states. This is especially true for the general Greenberger-Horne-Zeilinger (GHZ) states [7, 8]

$$
|\mathrm{GHZ}\rangle_{N}=\frac{1}{\sqrt{2}}\left(|0\rangle^{\otimes N}+|1\rangle^{\otimes N}\right)
$$

also known as cat states (see, e.g., [9]), which, according to most entanglement measures, are maximally entangled [6].

Various approaches to generating GHZ states have been proposed in the literature. A scheme for generating cat states of a single-mode optical field by means of conditional measurement was proposed in Ref. [10]. A one-step multi-atomic GHZ state generation in a non-resonant cavity by way of cavity-assisted collisions was considered in Ref. [11]. Ref. [12] discusses the possibility of generating GHZ states of electron spin qubits in a chain of quantum dots using the naturally available single-qubit rotations and the two-qubit Heisenberg exchange interaction. The minimum number of required operations in that proposal scales linearly with the number of qubits. In Ref. 13], in the context of capacitively coupled superconducting phase qubits, an $N$-qubit GHZ state is proposed to be generated by applying an initial one-qubit $\pi / 2$-pulse followed by an alternating sequence of $2(N-2)+1$ controlled-NOT (CNOT) and SWAP gates. In Ref. [14] it is shown how the GHZ state can be generated in a multi-qubit system by coupling it sequentially to a mesoscopic static completely mixed spin bath. In Ref. [15], in the context of circuit quantum electrodynamics, high-fidelity GHZ states (in a system whose mutual qubit detunings are large compared to the inter-qubit coupling strengths) are proposed to be probabilistically generated using one-qubit rotations and a single $N$-qubit dispersive readout.

In this paper we describe how GHZ states can be generated using a single $N$-qubit entangling pulse in a fully

*Electronic address: ag@physast.uga.edu

${ }^{\dagger}$ Electronic address: mcoffey@mines.edu 
connected network of qubits (see also [16]) interacting by way of anisotropic Heisenberg exchange [17]

$$
H_{\mathrm{int}}=\frac{1}{2} \sum_{\ell=1}^{N-1} \sum_{k=\ell+1}^{N}\left[g\left(\sigma_{x}^{\ell} \sigma_{x}^{k}+\sigma_{y}^{\ell} \sigma_{y}^{k}\right)+\tilde{g} \sigma_{z}^{\ell} \sigma_{z}^{k}\right],
$$

where $\sigma_{\mu}^{\ell}, \mu=x, y, z$, are the Pauli matrices $\sigma_{\mu}$ acting on the $\ell$-th qubit. We concentrate on the case $g \neq \tilde{g}$ only, which in the context of Josephson phase qubits (our initial motivation [18]) corresponds to either capacitive $(\tilde{g}=0$ ) or inductive $(0<|\tilde{g} / g|<0.1)$ coupling scheme. We show that the duration of the entangling pulse is given by

$$
t_{\mathrm{GHZ}}=\pi / 2|g-\tilde{g}|
$$

and thus in a typical experiment with $g \sim 10 \mathrm{MHz}$ the time $t_{\mathrm{GHZ}}$ is on the order of 25 ns, which is small compared to the usually achieved coherence times of $\sim 500 \mathrm{~ns}$ [19]. We point out that the interaction Hamiltonian given in Eq. (2) is the result of first projecting the exact system Hamiltonian onto the qubit subspace, switching to the interaction picture, and then applying the rotating wave approximation (RWA) in which the fast oscillating terms are ignored. Here, the time scale is set by a typical time to do a qubit operation, such as, e.g., the entangling time given above. The fast oscillations are the ones that occur at qubit transition frequencies, usually on the order of $10 \mathrm{GHz}$, and thus having transition times on the order of $0.1 \mathrm{~ns}$ [17].

Why employ fully connected networks?

If we adopt an important simplifying assumption of identical couplings on all qubit pairs, then the answer to the above question is: "symmetry, simplicity, and experimental practicality." As will be shown below, the symmetric coupling scheme allows a rigorous proof of the protocol for arbitrary $N$. The resulting GHZ sequence is, indeed, very simple: prepare the system in the uniform superposition state, turn the coupling on and wait for a given time $t_{\mathrm{GHZ}}$, then apply a final, corrective $N$-qubit rotation.

There is a nice experimental realization of fully connected networks involving capacitively coupled superconducting qubits proposed by Matthew Neeley and his collaborators at UCSB [20]. It consists of a superconducting island (an isolated piece of aluminum) connected via a capacitor to each of the qubits in the system. Using the standard methods of circuit analysis, one can transform this "star" configuration into an equivalent "delta" (or, fully connected network) configuration, in which there is a mutual capacitance between each pair of qubits. [The term "delta" comes from the three-qubit case, where the system looks like a triangle, a special case of the "star-delta" or "Y-delta" transform in electrical engineering [21].] It is then straightforward to show that $N$ qubits coupled to an island with capacitance $C_{\star}$ are equivalent to $N$ qubits coupled to each other in a complete graph with capacitance $C_{\Delta}=C_{\star} / N$ [20]. Thus, from the practical point of view, implementing a fully connected network using a solid state quantum computing architecture is relatively straightforward. There are still some residual errors in the coupling that are due to imperfections in the coupling capacitors, so the resulting pairwise couplings are not strictly identical. That somewhat limits applicability of the model and, in a rigorous analysis, must be taken into account. Below we show how the errors due to imperfections in the individual couplings can be drastically reduced by adjusting the entangling time and the final rotations in the $N=3,4$ cases.

Our final motivation for the use of fully connected networks comes from their applicability to problems in number theory. Two of us (MWC and RD) have recently proposed an effective way to perform primality testing using this model with a modest number of qubits [22].

We now turn to the description and the proof of the GHZ protocol itself.

\section{GHZ PROTOCOL}

First, notice that for any number of qubits $N$ the Hamiltonian (2) has eigenenergy (see Appendix A

$$
E_{\mathrm{GHZ}} \equiv \lambda_{0}(N)=C_{N}^{2}(\tilde{g} / 2)
$$

associated with the eigenstates $|0\rangle^{\otimes N}$ and $|1\rangle^{\otimes N}$, where $C_{N}^{2}=(N-1) N / 2$ is the total number of pairwise couplings present in the network. Then, the two families of the GHZ protocols are

$$
N=2,4,6, \ldots: \quad e^{-i \lambda_{0} t_{\mathrm{GHZ}}} e^{i(N / 2-1) \pi}|\mathrm{GHZ}\rangle_{N}=R_{3} R_{1} U_{\mathrm{ent}} R_{1}|0\rangle^{\otimes N},
$$

and

$$
N=3,5,7, \ldots: \quad e^{-i \lambda_{0} t_{\mathrm{GHZ}}} e^{i(-1)^{(N-3) / 2}(\pi / 4)}|\mathrm{GHZ}\rangle_{N}=R_{2} U_{\mathrm{ent}} R_{1}|0\rangle^{\otimes N}
$$


where

$$
R_{3}=e^{-i\left[2+(-1)^{N / 2}\right] \pi \sigma_{z}^{1} / 4}, \quad R_{1}=\otimes_{k=1}^{N} e^{-i \pi \sigma_{y}^{k} / 4}, \quad R_{2}=\otimes_{k=1}^{N} e^{-i \pi \sigma_{x}^{k} / 4},
$$

are the corresponding local rotations, and $U_{\mathrm{ent}}=e^{-i H_{\mathrm{int}} t_{\mathrm{GHZ}}}$ is the entangling pulse of duration $t_{\mathrm{GHZ}}=\pi / 2|g-\tilde{g}|$.

To see in detail how these protocols work we first re-write the fully uniform superposition state,

$$
|\psi\rangle_{\text {uniform }}=R_{1}|0\rangle^{\otimes N} \equiv \frac{1}{2^{N / 2}} \sum_{\xi_{k} \in\{0,1\}}\left|\xi_{1}\right\rangle \ldots\left|\xi_{N}\right\rangle,
$$

as a sum of partial uniform superpositions, each of which is characterized by the total number $j$ of up spins in the respective direct-product components, as follows:

$$
|\psi\rangle_{\text {uniform }}=\frac{1}{2^{N / 2}} \sum_{j=0}^{N} \sqrt{C_{N}^{j}}\left|W_{j}\right\rangle
$$

Here, $\left|W_{j}\right\rangle$ stands for a generalized $W$-state (with $j$ spins up), and $C_{N}^{j}=N ! / j !(N-j) !$ is the corresponding binomial expansion coefficient. For example, for $N=3,\left|W_{1}\right\rangle=[|001\rangle+|010\rangle+|100\rangle] / \sqrt{3},\left|W_{2}\right\rangle=[|011\rangle+|101\rangle+|110\rangle] / \sqrt{3}$, etc. Notice that for any $N$,

$$
\left[\left|W_{0}\right\rangle+\left|W_{N}\right\rangle\right] / \sqrt{2} \equiv\left[|0\rangle^{\otimes N}+|1\rangle^{\otimes N}\right] / \sqrt{2}=|\mathrm{GHZ}\rangle_{N} .
$$

We now state an important property of the interaction Hamiltonian $H_{\text {int }}$ :

The eigenvalue formula: For any $j=0,1,2, \ldots, N$, the states $\left|W_{j}\right\rangle$ are the eigenstates of $H_{\text {int }}$ associated with the eigenvalue

$$
\lambda_{j}(N)=j(N-j)(g-\tilde{g})+C_{N}^{2}(\tilde{g} / 2) .
$$

Proof outline. The operator $\Sigma_{z}=\sum_{k} \sigma_{z}^{k}$ commutes with $H_{\text {int }}$, providing a good quantum number, the total number of up spins in a given state. Since $H_{\text {int }}$ and $\Sigma_{z}$ share eigenvectors, the $\left|W_{j}\right\rangle$ are eigensubspaces. The proof of the analytical formula (11) for the eigenvalues is given in Appendix A Notice that since the Hamiltonian (2) commutes with the operator $X^{\otimes N}=\mathrm{NOT}^{\otimes N}$, if $\left|W_{j}\right\rangle$ is an eigenvector, then so is $\left|W_{N-j}\right\rangle$, with the same eigenvalue. This is a powerful property, as it immediately tells us that the eigenenergies are constrained to satisfy the relation $\lambda_{j}=\lambda_{N-j}$.

The eigenvalue formula immediately allows us to write down the effect of the entangling pulse on the uniform superposition,

$$
U_{\text {ent }}|\psi\rangle_{\text {uniform }}=\frac{1}{2^{N / 2}} \sum_{j=0}^{N} \sqrt{C_{N}^{j}} e^{-i \lambda_{j} t_{\mathrm{GHZ}}}\left|W_{j}\right\rangle,
$$

giving the state on which the final $N$-qubit rotations $R_{3} R_{1}, R_{2}$ will be acting. Assuming $g>\tilde{g}$, we have the useful relation,

$$
e^{-i \lambda_{j} t_{\mathrm{GHZ}}}=e^{-i \lambda_{0} t_{\mathrm{GHZ}}} \exp \left\{-i\left[j(N-j)(g-\tilde{g}) t_{\mathrm{GHZ}}\right]\right\}=e^{-i \lambda_{0} t_{\mathrm{GHZ}}} \exp [-i(\pi / 2) j(N-j)]=e^{-i \lambda_{0} t_{\mathrm{GHZ}}}(-i)^{j(N-j)} .
$$

Now, for $N$ odd (Appendix B),

$$
R_{2}^{-1}|\mathrm{GHZ}\rangle_{\mathrm{N} \text { odd }}=\frac{1}{2^{N / 2}} \sum_{j=0}^{N} \sqrt{C_{N}^{j}} \frac{i^{j}\left[1+(-1)^{j} i^{N}\right]}{\sqrt{2}}\left|W_{j}\right\rangle .
$$

In this case $i^{N}= \pm i$. Recalling that

$$
\left\langle W_{j} \mid W_{k}\right\rangle=\delta_{j k}
$$

we see that the full state is properly normalized. Combining Eqs. (12), (13), (14), with the identity

$$
\begin{aligned}
i^{j(N-j)} & =(-i)^{j(N-j)}=\cos [j(N-j) \pi / 2] \\
& =\exp \left[i(-1)^{(N+1) / 2} \frac{\pi}{4}\right] \frac{i^{j}}{\sqrt{2}}\left[1+(-1)^{j} i^{N}\right]=\exp \left[i(-1)^{(N-3) / 2} \frac{\pi}{4}\right] \frac{i^{j}\left[1+(-1)^{j} i^{N}\right]}{\sqrt{2}},
\end{aligned}
$$


gives the GHZ state protocol for odd $N$.

For $N$ even (Appendix C),

$$
R_{1}^{-1}\left[R_{3}\right]^{-1}|\mathrm{GHZ}\rangle_{\mathrm{N} \text { even }}=\frac{1}{2^{N / 2}} \sum_{j=0}^{N} \sqrt{C_{N}^{j}} \frac{(-1)^{j}+e^{-i \theta}}{\sqrt{2}}\left|W_{j}\right\rangle,
$$

where

$$
\theta(N)=(\pi / 2)\left[2+(-1)^{N / 2}\right]
$$

Combining Eqs. (12) and (17) with the identity

$$
(-i)^{j(N-j)}=e^{i \pi(N / 2-1)} \frac{e^{i \theta / 2}}{\sqrt{2}}\left[(-1)^{j}+e^{-i \theta}\right]
$$

gives the GHZ state protocol for even $N$. [When $g<\tilde{g}$, and $N$ is even, an extra factor of $e^{-i(\pi / 4)\left(\sigma_{z}^{2}+\sigma_{z}^{3}\right)}$ should be inserted after $R_{3}$, otherwise the GHZ state accumulates an internal phase of (-1).]

Notice that for $g=\tilde{g}$, the uniform superposition state is an eigenstate of the interaction Hamiltonian $H_{\text {int }}$. Thus, the $|\psi\rangle_{\text {uniform }}$ does not change (apart from accumulating an overall phase) under the action of the entangling pulse $U_{\text {ent }}$. This explains the previously stated requirement $g \neq \tilde{g}$ (cf. [17]).

\section{FIDELITY DEGRADATION DUE TO COUPLING ERRORS}

Achieving identical couplings on all qubit pairs is experimentally difficult. One way to reduce errors due to coupling imperfections is to adjust the final qubit rotations and the entangling time in such a way as to maximize the fidelity of the resulting state. Figure 1 shows the result of numerical optimization with respect to the Frobenius distance between the generated state and the GHZ target,

$$
\mathcal{F}=1-\sqrt{\left(\left\langle\left.\psi\right|_{\text {opt }}-\langle\mathrm{GHZ}|\right)\left(|\psi\rangle_{\mathrm{opt}}-|\mathrm{GHZ}\rangle\right)\right.},
$$

for $N=3$. Here the Hamiltonian is given by

$$
H_{\mathrm{int}}=\left(g_{12} / 2\right)\left[\left(\sigma_{x}^{1} \sigma_{x}^{2}+\sigma_{y}^{1} \sigma_{y}^{2}\right)+\left(1-\eta_{23}\right)\left(\sigma_{x}^{2} \sigma_{x}^{3}+\sigma_{y}^{2} \sigma_{y}^{3}\right)+\left(1-\eta_{13}\right)\left(\sigma_{x}^{1} \sigma_{x}^{3}+\sigma_{y}^{1} \sigma_{y}^{3}\right)+\kappa\left(\sigma_{z}^{1} \sigma_{z}^{2}+\sigma_{z}^{2} \sigma_{z}^{3}+\sigma_{z}^{1} \sigma_{z}^{3}\right)\right],
$$

where $g_{12}>0$ is the reference coupling on qubits 1 and $2, \eta_{23}, \eta_{13} \geq 0$ are the coupling errors on pairs 23 and 13 , and $\kappa$ is the $Z Z$ coupling, which for simplicity is assumed to be constant throughout the network (the results of this section do not depend on this assumption). Our simulations indicate that the best fidelity is achieved when the initial pulse is chosen to generate the uniform superposition state, similar to how it is done in the ideal case with $\eta_{23}=\eta_{13}=0$. A typical optimized state, here shown at $\kappa=0.05, \eta_{23}=0.02$, and $\eta_{13}=0.06$, has the form

$$
|\psi\rangle_{\mathrm{opt}}=\left(\begin{array}{c}
0.707099 \\
0.000692 \\
-0.001956 \\
0.002566 \\
0.002566 \\
-0.001956 \\
0.000692 \\
0.707099
\end{array}\right)
$$

which corresponds to $t_{\mathrm{opt}} /\left(\pi / 2 g_{12}(1-\kappa)\right)=1.0505, \alpha_{\mathrm{opt}}^{1,2,3} /(\pi / 2)=0.9785,0.9713,0.9825$, and $\mathcal{F}=0.9953$. This may be compared to the non-corrected state,

$$
|\psi\rangle_{\text {original }}=\left(\begin{array}{c}
0.706616 \\
0.000697+0.015431 i \\
-0.002792+0.010929 i \\
0.002988+0.017844 i \\
0.002988+0.017844 i \\
-0.002792+0.010929 i \\
0.000697+0.015431 i \\
0.706616
\end{array}\right)
$$



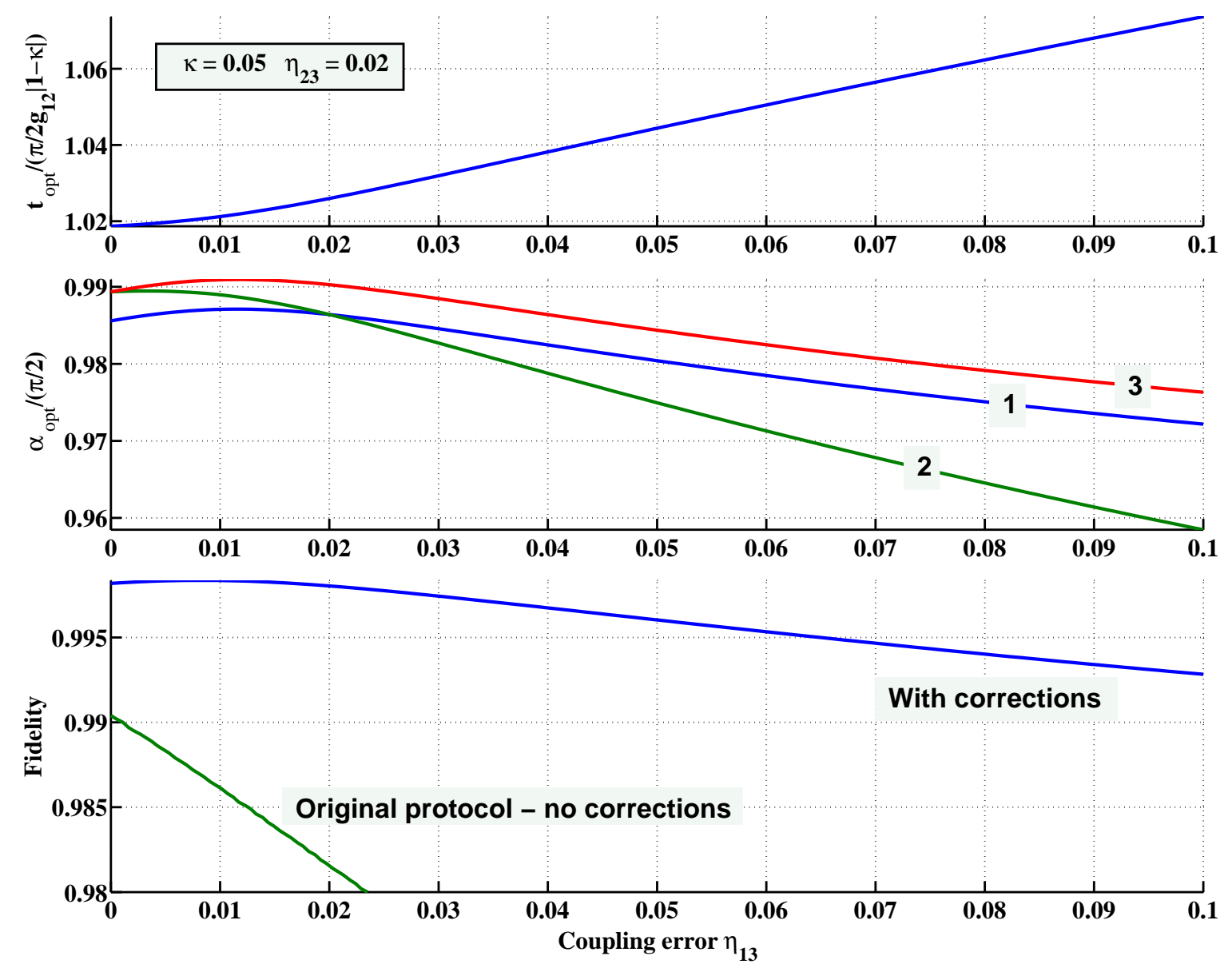

FIG. 1: (Color online) Optimal values of the final rotation angles $\alpha_{\mathrm{opt}}^{i}, i=1,2,3$, and the entangling time $t_{\mathrm{opt}}$ that minimize the error due to coupling imperfections in the $N=3$ case. The gate fidelity is defined in Eq. (20). The corresponding Hamiltonian is given in Eq. (21). Here, $\kappa=0.05, \eta_{23}=0.02$, and $0 \leq \eta_{13} \leq 0.10$. Fidelity curve for the original protocol run with no corrections is also shown.

that has $\mathcal{F}=0.9628$, which we get by running the original protocol at the same values of $\kappa$ and $\eta_{23}, \eta_{13}$.

We have also performed optimization in the $N=4$ case (not shown) where it was found that in order to maximize the fidelity of the resulting state only the entangling time $t_{\mathrm{GHZ}}$ and the final $Z$-rotation on the first qubit have to be adjusted. The $R_{1}$ pulse appearing on both sides of $U_{\text {ent }}$ should, however, remain the same.

The following argument indicates that similar optimization results may be expected for arbitrary $N$. Assuming coupling errors in the interaction Hamiltonian to be sufficiently small, their effect is to perturb (in general all of) the $N+1$ eigenvalues $\lambda_{j}$ corresponding to the $\left|W_{j}\right\rangle$ states. If we then optimize the fidelity on the evolution time $t_{\mathrm{GHZ}}$ and $N$ final single-qubit rotation angles, we have $N+1$ parameters to do so. We thus have $N+1$ parameters in which to correct $N+1$ state coefficient errors due to $N+1$ perturbed phases coming from the perturbed eigenvalues. Minimizing the Frobenius distance needed for the fidelity is essentially solving a nonlinear least squares problem. Under only weak conditions, we will have a solution guaranteed as we have $N+1$ parameters at our disposal. If there were errors in the initial fully uniform superposition state, then the $N+1$ parameters would be insufficient in number to correct errors from both the initial state and a perturbed Hamiltonian. Improvements would be possible, but the correct initial state would then seem to be the best place to start from when precisely $N+1$ parameters are available to later correct errors in the protocol. This argument shows that finding corrected fidelity by adjusting the entangling time and the final $N$ rotation angles is always possible. It does not state how well the correction can be done, but the $N=3$ and $N=4$ cases presented above give illustrations. 


\section{CONCLUSION}

In summary, we have demonstrated how the single-step GHZ protocol proposed for triangularly coupled superconducting qubit system can be extended to a fully connected network of an arbitrary number of qubits. Two types of protocols can be identified here, depending on whether $N$ is odd or even. When $N$ is odd, the protocol (up to an overall insignificant phase) is the same as in the three-qubit case; it is given by a generic sequence $X_{\pi / 2} U_{\text {ent }} Y_{\pi / 2}|0\rangle{ }^{\otimes N}$, with $X_{\pi / 2}$ and $Y_{\pi / 2}$ representing the corresponding $x$ and $y$ rotations performed on the $N$ qubits. When $N$ is even, the final rotation gets modified and the sequence becomes $Z_{\theta}^{1} Y_{\pi / 2} U_{\text {ent }} Y_{\pi / 2}|0\rangle^{\otimes N}$, where $Z_{\theta}^{1}$ is a $z$-rotation on the first qubit by angle $\theta$ which is given in Eq. (18). As a subproblem to describe the dynamics in the $\left|W_{j}\right\rangle$ subspaces, we exactly solved for the corresponding eigenenergies.

We emphasize again that implementing identical couplings on all pairs of qubits is a difficult task. Here we proposed a possible approach to reducing the errors due to coupling imperfections in the experimentally important low-dimensional cases.

\section{APPENDIX A: PROOF OF THE EIGENVALUE FORMULA, EQ. (11).}

\section{Raising and lowering operators}

For the purposes of this Appendix the matrix representations of states $|0\rangle=(0,1)^{\mathrm{T}}$ and $|1\rangle=(1,0)^{\mathrm{T}}$ are taken. We first define the raising and lowering operators $S_{ \pm}^{k}=\frac{1}{2}\left(\sigma_{x}^{k} \pm i \sigma_{y}^{k}\right)$ with the following properties:

$$
\begin{gathered}
S_{-}|0\rangle=0, S_{+}|1\rangle=0,\left(S_{-}\right)^{2}=\left(S_{+}\right)^{2}=0, S_{+}|0\rangle=|1\rangle, S_{-}|1\rangle=|0\rangle, \\
\sigma_{z}^{k} S_{+}^{k}=S_{+}^{k}, \sigma_{z}^{k} S_{-}^{k}=S_{-}^{k}, S_{+}^{k} \sigma_{z}^{k}=-S_{+}^{k}, S_{-}^{k} \sigma_{z}^{k}=S_{-}^{k}, \\
\sigma_{z}^{k}=\mathbf{I}_{2}-2 S_{-}^{k} S_{+}^{k}, \sigma_{z}|0\rangle=-|0\rangle, \sigma_{z}|1\rangle=|1\rangle,
\end{gathered}
$$

and

$$
\left[S_{+}^{k_{1}}, S_{-}^{k_{2}}\right]=\delta_{k_{1} k_{2}} \sigma_{z}^{k_{2}}, \quad\left[\sigma_{z}^{k_{1}}, S_{ \pm}^{k_{2}}\right]= \pm 2 \delta_{k_{1} k_{2}} S_{ \pm}^{k_{2}},
$$

It will be useful to define the operators

$$
\Sigma_{ \pm}=\sum_{k=1}^{N} S_{ \pm}^{k}, \quad \Sigma_{z}=\sum_{k=1}^{N} \sigma_{z}^{k}
$$

obeying

$$
\left[\Sigma_{+}, \Sigma_{-}\right]=\Sigma_{z}, \quad\left[\Sigma_{z}, \Sigma_{ \pm}\right]= \pm 2 \Sigma_{ \pm} .
$$

Using these commutation relations we arrive at the following useful products.

Lemma 1:

$$
\Sigma_{z}\left(\Sigma_{ \pm}\right)^{j}=\left(\Sigma_{ \pm}\right)^{j} \Sigma_{z} \pm 2 j\left(\Sigma_{ \pm}\right)^{j} .
$$

Proof (by induction). For $j=1$,

$$
\left[\Sigma_{z}, \Sigma_{ \pm}\right]=\Sigma_{z} \Sigma_{ \pm}-\Sigma_{ \pm} \Sigma_{z}= \pm 2 \Sigma_{ \pm}, \quad \Sigma_{z} \Sigma_{ \pm}=\Sigma_{ \pm} \Sigma_{z} \pm 2 \Sigma_{ \pm} .
$$

Assuming that $\Sigma_{z}\left(\Sigma_{ \pm}\right)^{n}=\left(\Sigma_{ \pm}\right)^{n} \Sigma_{z} \pm 2 n\left(\Sigma_{ \pm}\right)^{n}$, we have

$$
\begin{aligned}
\Sigma_{z}\left(\Sigma_{ \pm}\right)^{n+1} & =\left(\Sigma_{z}\left(\Sigma_{ \pm}\right)^{n}\right) \Sigma_{ \pm} \\
& =\left(\left(\Sigma_{ \pm}\right)^{n} \Sigma_{z} \pm 2 n\left(\Sigma_{ \pm}\right)^{n}\right) \Sigma_{ \pm} \\
& =\left(\Sigma_{ \pm}\right)^{n}\left(\Sigma_{ \pm} \Sigma_{z} \pm 2 \Sigma_{ \pm}\right) \pm 2 n\left(\Sigma_{ \pm}\right)^{n+1} \\
& =\left(\Sigma_{ \pm}\right)^{n+1} \Sigma_{z} \pm 2(n+1)\left(\Sigma_{ \pm}\right)^{n+1}
\end{aligned}
$$

Lemma 2:

$$
\Sigma_{-}\left(\Sigma_{+}\right)^{j}=\left(\Sigma_{+}\right)^{j} \Sigma_{-}-j\left(\Sigma_{+}\right)^{j-1} \Sigma_{z}-j(j-1)\left(\Sigma_{+}\right)^{j-1} .
$$


Proof (by induction). For $j=1$,

$$
\left[\Sigma_{+}, \Sigma_{-}\right]=\Sigma_{+} \Sigma_{-}-\Sigma_{-} \Sigma_{+}=\Sigma_{z}, \quad \Sigma_{-} \Sigma_{+}=\Sigma_{+} \Sigma_{-}-\Sigma_{z} .
$$

For $j=2$,

$$
\begin{aligned}
\Sigma_{-} \Sigma_{+} \Sigma_{+} & =\left(\Sigma_{+} \Sigma_{-}-\Sigma_{z}\right) \Sigma_{+} \\
& =\Sigma_{+}\left(\Sigma_{+} \Sigma_{-}-\Sigma_{z}\right)-\Sigma_{+} \Sigma_{z}-2 \Sigma_{+} \\
& =\left(\Sigma_{+}\right)^{2} \Sigma_{-}-2 \Sigma_{+} \Sigma_{z}-2 \Sigma_{+} .
\end{aligned}
$$

Now, assuming $\Sigma_{-}\left(\Sigma_{+}\right)^{n}=\left(\Sigma_{+}\right)^{n} \Sigma_{-}-n\left(\Sigma_{+}\right)^{n-1} \Sigma_{z}-n(n-1)\left(\Sigma_{+}\right)^{n-1}$, we get

$$
\begin{aligned}
\Sigma_{-}\left(\Sigma_{+}\right)^{n+1} & =\left(\Sigma_{-}\left(\Sigma_{+}\right)^{n}\right) \Sigma_{+} \\
& =\left(\Sigma_{+}\right)^{n}\left(\Sigma_{-} \Sigma_{+}\right)-n\left(\Sigma_{+}\right)^{n-1}\left(\Sigma_{z} \Sigma_{+}\right)-n(n-1)\left(\Sigma_{+}\right)^{n} \\
& =\left(\Sigma_{+}\right)^{n+1} \Sigma_{-}-\left(\Sigma_{+}\right)^{n} \Sigma_{z}-n\left(\Sigma_{+}\right)^{n} \Sigma_{z}-2 n\left(\Sigma_{+}\right)^{n}-n(n-1)\left(\Sigma_{+}\right)^{n} \\
& =\left(\Sigma_{+}\right)^{n+1} \Sigma_{-}-(n+1)\left(\Sigma_{+}\right)^{n} \Sigma_{z}-(n(n-1)+2 n)\left(\Sigma_{+}\right)^{n} \\
& =\left(\Sigma_{+}\right)^{n+1} \Sigma_{-}-(n+1)\left(\Sigma_{+}\right)^{n} \Sigma_{z}-((n+1)((n+1)-1))\left(\Sigma_{+}\right)^{n} .
\end{aligned}
$$

We now generate partial uniform superpositions (generalized $W$ states) using the raising and lowering operators:

$$
\begin{aligned}
\left|W_{0}\right\rangle & =1|0\rangle^{\otimes N} \\
\left|W_{1}\right\rangle & =\frac{1}{\sqrt{C_{N}^{1}}} \sum_{k=1}^{N} S_{+}^{k}|0\rangle^{\otimes N} \\
\left|W_{2}\right\rangle & =\frac{1}{\sqrt{C_{N}^{2}}} \sum_{k_{1}=1}^{N-1} \sum_{k_{2}>k_{1}}^{N} S_{+}^{k_{1}} S_{+}^{k_{2}}|0\rangle^{\otimes N} \\
\vdots & \\
\left|W_{j}\right\rangle & =\frac{1}{\sqrt{C_{N}^{j}}} \sum_{k_{1}=1}^{N-j+1} \sum_{k_{2}>k_{1}}^{N-j+2} \sum_{k_{j-1}>k_{j-2}}^{N-1} \sum_{k_{j}>k_{j-1}}^{N} S_{+}^{k_{1}} S_{+}^{k_{2}} \ldots S_{+}^{k_{j-1}} S_{+}^{k_{j}}|0\rangle^{\otimes N} .
\end{aligned}
$$

If we take into account all double counting we can see that these states can also be written in a form

$$
\begin{aligned}
\left|W_{0}\right\rangle & =1|0\rangle^{\otimes N} \\
\left|W_{1}\right\rangle & =\frac{1}{\sqrt{C_{N}^{1}}} \sum_{k=1}^{N} S_{+}^{k}|0\rangle^{\otimes N}=\frac{1}{\sqrt{C_{N}^{1}}} \Sigma_{+}|0\rangle^{\otimes N} \\
\left|W_{2}\right\rangle & =\frac{1}{2 ! \sqrt{C_{N}^{2}}} \sum_{k_{1}=1}^{N} \sum_{k_{2}=1}^{N} S_{+}^{k_{1}} S_{+}^{k_{2}}|0\rangle^{\otimes N}=\frac{1}{2 ! \sqrt{C_{N}^{2}}}\left(\Sigma_{+}\right)^{2}|0\rangle^{\otimes N}, \\
& \vdots \\
\left|W_{j}\right\rangle & =\frac{1}{j ! \sqrt{C_{N}^{j}}} \sum_{k_{1}=1}^{N} \sum_{k_{2}=1}^{N} \ldots \sum_{k_{j-1}=1}^{N} \sum_{k_{j}=1}^{N} S_{+}^{k_{1}} S_{+}^{k_{2}} \ldots S_{+}^{k_{j-1}} S_{+}^{k_{j}}|0\rangle^{\otimes N}=\frac{1}{j ! \sqrt{C_{N}^{j}}}\left(\Sigma_{+}\right)^{j}|0\rangle^{\otimes N}
\end{aligned}
$$

Thus,

$$
\begin{aligned}
\Sigma_{+}\left|W_{j}\right\rangle & =\frac{1}{j ! \sqrt{C_{N}^{j}}}\left(\Sigma_{+}\right)^{j+1}|0\rangle^{\otimes N} \\
& =\frac{1}{j ! \sqrt{C_{N}^{j}}}\left((j+1) ! \sqrt{C_{N}^{j+1}}\right)\left|W_{j+1}\right\rangle, \\
& =\sqrt{(N-j)(j+1)}\left|W_{j+1}\right\rangle .
\end{aligned}
$$


Using the lemmas and the facts that $\Sigma_{-}|0\rangle^{\otimes N}=0$ and $\Sigma_{z}|0\rangle^{\otimes N}=-N|0\rangle^{\otimes N}$, we find:

$$
\begin{aligned}
\Sigma_{-}\left|W_{j}\right\rangle & =\frac{1}{j ! \sqrt{C_{N}^{j}}}\left(\Sigma_{-}\left(\Sigma_{+}\right)^{j}\right)|0\rangle^{\otimes N}, \\
& =\frac{1}{j ! \sqrt{C_{N}^{j}}}\left(\left(\Sigma_{+}\right)^{j} \Sigma_{-}-j\left(\Sigma_{+}\right)^{j-1} \Sigma_{z}-j(j-1)\left(\Sigma_{+}\right)^{j-1}\right)|0\rangle^{\otimes N}, \\
& =\frac{j(N-j+1)}{j ! \sqrt{C_{N}^{j}}}\left(\Sigma_{+}\right)^{j-1}|0\rangle^{\otimes N}, \\
& =\sqrt{j(N-j+1)}\left|W_{j-1}\right\rangle,
\end{aligned}
$$

and

$$
\begin{aligned}
\Sigma_{z}\left|W_{j}\right\rangle & =\frac{1}{j ! \sqrt{C_{N}^{j}}} \Sigma_{z}\left(\Sigma_{+}\right)^{j}|0\rangle^{\otimes N}, \\
& =\frac{1}{j ! \sqrt{C_{N}^{j}}}\left(\left(\Sigma_{+}\right)^{j} \Sigma_{z}+2 j\left(\Sigma_{+}\right)^{j}\right)|0\rangle^{\otimes N}, \\
& =\frac{1}{j ! \sqrt{C_{N}^{j}}}(-N+2 j)\left(\Sigma_{+}\right)^{j}|0\rangle^{\otimes N}, \\
& =(2 j-N)\left|W_{j}\right\rangle .
\end{aligned}
$$

\section{Eigenvalues of $H_{\mathrm{int}}$}

First notice that

$$
\sigma_{x}^{k_{1}} \sigma_{x}^{k_{2}}+\sigma_{y}^{k_{1}} \sigma_{y}^{k_{2}}=2\left(S_{+}^{k_{1}} S_{-}^{k_{2}}+S_{-}^{k_{1}} S_{+}^{k_{2}}\right)
$$

from which it follows that

$$
H_{\mathrm{int}}=\frac{1}{2} \sum_{k_{1}=1}^{N-1} \sum_{k_{2}=k_{1}+1}^{N}\left(2 g\left(S_{+}^{k_{1}} S_{-}^{k_{2}}+S_{-}^{k_{1}} S_{+}^{k_{2}}\right)+g_{z} \sigma_{z}^{k_{1}} \sigma_{z}^{k_{2}}\right) .
$$

Also,

$$
\Sigma_{z} \Sigma_{z}=\sum_{k_{1}, k_{2}=1}^{N} \sigma_{z}^{k_{1}} \sigma_{z}^{k_{2}}=\sum_{k_{1} \neq k_{2}=1}^{N} \sigma_{z}^{k_{1}} \sigma_{z}^{k_{2}}+\sum_{k=1}^{N}\left(\sigma_{z}^{k}\right)^{2}=2 \sum_{k_{1}=1}^{N-1} \sum_{k_{2}=k_{1}+1}^{N} \sigma_{z}^{k_{1}} \sigma_{z}^{k_{2}}+N \mathbf{I},
$$

and similarly,

$$
\begin{aligned}
& \Sigma_{+} \Sigma_{-}=\sum_{k_{1}, k_{2}=1}^{N} S_{+}^{k_{1}} S_{-}^{k_{2}}=\sum_{k_{1} \neq k_{2}=1}^{N} S_{+}^{k_{1}} S_{-}^{k_{2}}+\sum_{k=1}^{N} S_{+}^{k} S_{-}^{k}=2 \sum_{k_{1}=1}^{N-1} \sum_{k_{2}=k_{1}+1}^{N} S_{+}^{k_{1}} S_{-}^{k_{2}}+\sum_{k=1}^{N} S_{+}^{k} S_{-}^{k}, \\
& \Sigma_{-} \Sigma_{+}=\sum_{k_{1}, k_{2}=1}^{N} S_{-}^{k_{1}} S_{+}^{k_{2}}=\sum_{k_{1} \neq k_{2}=1}^{N} S_{-}^{k_{1}} S_{+}^{k_{2}}+\sum_{k=1}^{N} S_{-}^{k} S_{+}^{k}=2 \sum_{k_{1}=1}^{N-1} \sum_{k_{2}=k_{1}+1}^{N} S_{-}^{k_{1}} S_{+}^{k_{2}}+\sum_{k=1}^{N} S_{-}^{k} S_{+}^{k} .
\end{aligned}
$$

Using the fact that $\sum_{k=1}^{N} S_{+}^{k} S_{-}^{k}+\sum_{k=1}^{N} S_{-}^{k} S_{+}^{k}=N \mathbf{I}$, we re-write $H_{\text {int }}$ in the form

$$
\begin{aligned}
H_{\mathrm{int}} & =\frac{1}{2}\left(2 g \sum_{k_{1}=1}^{N-1} \sum_{k_{2}=k_{1}+1}^{N} S_{+}^{k_{1}} S_{-}^{k_{2}}+2 g \sum_{k_{1}=1}^{N-1} \sum_{k_{2}=k_{1}+1}^{N} S_{+}^{k_{2}} S_{-}^{k_{1}}+\tilde{g} \sum_{k_{1}=1}^{N-1} \sum_{k_{2}=k_{1}+1}^{N} \sigma_{z}^{k_{1}} \tilde{g}^{k_{2}}\right) \\
& =\frac{1}{2}\left(2 g\left(\frac{1}{2}\left(\Sigma_{+} \Sigma_{-}-\sum_{k=1}^{N} S_{+}^{k} S_{-}^{k}\right)\right)+2 g\left(\frac{1}{2}\left(\Sigma_{-} \Sigma_{+}-\sum_{k=1}^{N} S_{-}^{k} S_{+}^{k}\right)\right)+\tilde{g}\left(\frac{1}{2}\left(\Sigma_{z} \Sigma_{z}-N \mathbf{I}\right)\right)\right), \\
& =\frac{1}{4}\left(2 g\left(\Sigma_{+} \Sigma_{-}+\Sigma_{-} \Sigma_{+}\right)+\tilde{g} \Sigma_{z} \Sigma_{z}-(2 g+\tilde{g}) N \mathbf{I}\right),
\end{aligned}
$$


or

$$
H_{\mathrm{int}}=\frac{1}{4}\left(2 g H_{\mathrm{int}}^{g}+\tilde{g} H_{\mathrm{int}}^{\tilde{g}}\right)
$$

where

$$
H_{\mathrm{int}}^{g}=\Sigma_{+} \Sigma_{-}+\Sigma_{-} \Sigma_{+}-N \mathbf{I}, \quad H_{\mathrm{int}}^{\tilde{g}}=\Sigma_{z} \Sigma_{z}-N \mathbf{I} .
$$

We now act with $H_{\text {int }}^{g}$ on the partial uniform states,

$$
\begin{aligned}
H_{\mathrm{int}}^{g}\left|W_{j}\right\rangle & =\left(\Sigma_{+} \Sigma_{-}+\Sigma_{-} \Sigma_{+}-N \mathbf{I}\right)\left|W_{j}\right\rangle, \\
& =\sqrt{j(N-j+1)} \Sigma_{+}\left|W_{j-1}\right\rangle+\sqrt{(N-j)(j+1)} \Sigma_{-}\left|W_{j+1}\right\rangle-N\left|W_{j}\right\rangle, \\
& =(j(N-j+1)+(N-j)(j+1)-N)\left|W_{j}\right\rangle, \\
& =2 j(N-j)\left|W_{j}\right\rangle,
\end{aligned}
$$

and similarly,

$$
H_{\mathrm{int}}^{\tilde{g}}\left|W_{j}\right\rangle=\left(\Sigma_{z} \Sigma_{z}-N \mathbf{I}\right)\left|W_{j}\right\rangle=\left((2 j-N)^{2}-N\right)\left|W_{j}\right\rangle
$$

It is then easy to see that $H_{\text {int }}\left|W_{j}\right\rangle=\lambda_{j}(N)\left|W_{j}\right\rangle$, where

$$
\lambda_{j}(N)=\frac{1}{4}\left(4 j(N-j) g+\left((2 j-N)^{2}-N\right) \tilde{g}\right)=j(N-j)(g-\tilde{g})+C_{N}^{2}(\tilde{g} / 2) .
$$

This completes the proof of the eigenvalue formula.

\section{APPENDIX B: PROOF OF EQ. (14)}

Here, $N$ is odd. Using the fact that for $y \in\{0,1\}$,

$$
e^{-i \pi \sigma_{x} / 4}|y\rangle=\frac{(-i)^{y}}{\sqrt{2}}\left[|0\rangle-i(-1)^{y}|1\rangle\right]=\frac{(-i)^{y}}{\sqrt{2}} \sum_{z \in\{0,1\}}(-i)^{z}(-1)^{y z}|z\rangle,
$$

we see that the action of $R_{2}^{-1}$ on arbitrary direct-product states is given by

$$
R_{2}^{-1}\left|y_{1} y_{2} \ldots y_{N}\right\rangle=\frac{i^{y_{1}+y_{2}+\ldots+y_{N}}}{2^{N / 2}} \sum_{z_{k} \in\{0,1\}} i^{z_{1}+z_{2}+\ldots+z_{N}}(-1)^{y_{1} z_{1}+y_{2} z_{2}+\ldots+y_{N} z_{N}}\left|z_{1}\right\rangle\left|z_{2}\right\rangle \ldots\left|z_{N}\right\rangle \text {. }
$$

We now let $z_{\text {tot }}=\sum_{k=1}^{N} z_{k}$ when occurring in such sums, and write more compactly

$$
R_{2}^{-1}\left|y_{1} y_{2} \ldots y_{N}\right\rangle=\frac{i^{y_{1}+y_{2}+\ldots+y_{N}}}{2^{N / 2}} \sum_{z_{k} \in\{0,1\}} i^{z_{\text {tot }}}(-1)^{y \cdot z}\left|z_{1}\right\rangle\left|z_{2}\right\rangle \ldots\left|z_{N}\right\rangle .
$$

We note that $0 \leq z_{\text {tot }} \leq N$. We then have

$$
R_{2}^{-1}|0\rangle^{\otimes N}=\frac{1}{2^{N / 2}} \sum_{z_{k}} i^{z_{t o t}}\left|z_{1}\right\rangle \ldots\left|z_{N}\right\rangle, \quad R_{2}^{-1}|1\rangle^{\otimes N}=\frac{i^{N}}{2^{N / 2}} \sum_{z_{j}} i^{z_{\text {tot }}}(-1)^{z_{\text {tot }}}\left|z_{1}\right\rangle \ldots\left|z_{N}\right\rangle .
$$

Therefore,

$$
R_{2}^{-1}|\mathrm{GHZ}\rangle_{\mathrm{N} \text { odd }}=R_{2}^{-1} \frac{1}{\sqrt{2}}\left(|0\rangle^{\otimes N}+|1\rangle^{\otimes N}\right)=\frac{1}{\sqrt{2} 2^{N / 2}} \sum_{z_{j}} i^{z_{\text {tot }}}\left[1+(-1)^{z_{\text {tot }}} i^{N}\right]\left|z_{1}\right\rangle \ldots\left|z_{N}\right\rangle .
$$

Replacing the sum over $z_{k}$ 's by $z_{\text {tot }}$, which is just an integer $j$ in the range $0, \ldots, N$, gives

$$
R_{2}^{-1}|\mathrm{GHZ}\rangle_{\mathrm{N} \text { odd }}=\frac{1}{2^{N / 2}} \sum_{j=0}^{N} \sqrt{C_{N}^{j}} \frac{i^{j}\left[1+(-1)^{j} i^{N}\right]}{\sqrt{2}}\left|W_{j}\right\rangle
$$




\section{APPENDIX C: PROOF OF EQ. (17)}

Here, $N$ is even. Note that for $x \in\{0,1\}$,

$$
e^{-i \pi \sigma_{y} / 4}|x\rangle=\frac{1}{\sqrt{2}}\left[(-1)^{x}|0\rangle+|1\rangle\right]=\frac{1}{\sqrt{2}} \sum_{z \in\{0,1\}}(-1)^{x(z-1)}|z\rangle,
$$

which gives

$$
R_{1}^{-1}|x\rangle=\frac{1}{2^{N / 2}} \sum_{z_{j} \in\{0,1\}}(-1)^{x \cdot z}(-1)^{z_{t o t}}\left|z_{1}\right\rangle\left|z_{2}\right\rangle \ldots\left|z_{N}\right\rangle
$$

Since

$$
R_{1}^{-1}|0\rangle^{\otimes N}=\frac{1}{2^{N / 2}} \sum_{z_{j}}(-1)^{z_{\text {tot }}}\left|z_{1}\right\rangle \ldots\left|z_{N}\right\rangle, \quad R_{1}^{-1}|1\rangle^{\otimes N}=\frac{1}{2^{N / 2}} \sum_{z_{j}}\left|z_{1}\right\rangle \ldots\left|z_{N}\right\rangle
$$

we have

$$
\begin{aligned}
R_{1}^{-1}\left[R_{3}\right]^{-1}|\mathrm{GHZ}\rangle_{\mathrm{N} \text { even }} & =R_{1}^{-1} \frac{1}{\sqrt{2}}\left(|0\rangle^{\otimes N}+e^{i \varphi}|1\rangle^{\otimes N}\right) \\
& =\frac{1}{\sqrt{2} 2^{N / 2}} \sum_{z_{j}}\left[(-1)^{z_{t o t}}+e^{i \varphi}\right] \sum_{z_{j}}\left|z_{1}\right\rangle \ldots\left|z_{N}\right\rangle \\
& =\frac{1}{2^{N / 2}} \sum_{j=0}^{N} \sqrt{C_{N}^{j}} \frac{\left[(-1)^{j}+e^{i \varphi}\right]}{\sqrt{2}}\left|W_{j}\right\rangle,
\end{aligned}
$$

where $\varphi=-\theta(N)$.

\section{ACKNOWLEDGMENTS}

The work of AG was supported by IARPA under grant W911NF-08-1-0336 and by the NSF under grant CMS0404031. The work of MWC and RD was supported in part by a grant from Northrop Grumman. We thank Sahel Ashhab, John Martinis, and Matthew Neeley for useful discussions.

[1] A. Peres and D. Terno, Rev. Mod. Phys. 76, 93 (2004).

[2] M. Hillery, V. Buzek, and A. Berthiaume, Phys. Rev. A 59, 1829 (1999).

[3] Z. Zhao, et. al. Nature 430, 54 (2004).

[4] Shor, P. W. in Proc. 37th Symp. on the Foundations of Computer Science (FOCS), p. 56, IEEE Press, Los Alamitos, California (1996).

[5] E. Knill, Nature 434, 39 (2005).

[6] M. A. Nielsen and I. L. Chuang, Quantum Computation and Quantum Information, Cambridge University Press (2000).

[7] D. M. Greenberger, M. Horne, and A. Zeilinger, in Bell's Theorem, Quantum Theory, and Conceptions of the Universe, ed. M. Kafatos, p. 73, Kluwer Academic, Dordrecht (1989).

[8] D. M. Greenberger, M. Horne, A. Shimony, and A. Zeilinger, Am. J. Phys. 113158 (1990).

[9] D. Leibfried, E. Knill, S. Seidelin, J. Britton, R. B. Blakestad, J. Chiaverini, D. B. Hume, W. M. Itano, J. D. Jost, C. Langer, R. Ozeri, R. Reichle and D. J. Wineland, Nature 438, 639 (2005).

[10] M. Dakna, T. Anhut, T. Opatrný, L. Knöll, and D.-G. Welsch, Phys. Rev. A 55, 3184 (1997).

[11] S.-B. Zheng, Phys. Rev. Lett 87, 230404 (2001).

[12] F. Bodoky and M. Blaauboer, Phys. Rev. A 76, 052309 (2007).

[13] Sh. Matsuo, S. Ashhab, T. Fujii, F. Nori, K. Nagai, and N. Hatakenaka, J. Phys. Soc. Jap 76, 054802 (2007).

[14] H. Christ, J. I. Cirac, and G. Giedke, Phys. Rev. B 78, 125314 (2008).

[15] L. S. Bishop, L. Tornberg, D. Price, E. Ginossar, A. Nunnenkamp, A. A. Houck, J. M. Gambetta, J. Koch, G. Johansson, S. M. Girvin and R. J. Schoelkopf, New. J. Phys. 11, 073040 (2009).

[16] D. I. Tsomokos, S. Ashhab and F. Nori, New J. Phys. 10, 113020 (2008). 
[17] A. Galiautdinov, J. M. Martinis, Phys. Rev. A 78, 010305(R) (2008).

[18] M. Steffen, M. Ansmann, R. C. Bialczak, N. Katz, E. Lucero, R. McDermott, M. Neeley, E. M. Weig, A. N. Cleland, J. M. Martinis, Science 313, 1423 (2006).

[19] J. M. Martinis, Q. Inf. Proc. 8, 81 (2009).

[20] M. Neeley, private communication.

[21] See, e.g., P. Lorrain, D. R. Corson, F. Lorrain, Electromagnetic Fields and Waves, 3rd ed., p. 163, W. H. Freeman and Company, New York (1988).

[22] M. W. Coffey and R. Deiotte, in preparation (2009). 